\title{
Studying the influence of object size on the range of distance measurement in the new Depth From Defocus method
}

\author{
Krzysztof Murawski \\ Military University of Technology \\ ul. Kaliskiego 2, \\ 01-489 Warsaw, Poland, \\ IEEE Member \# 92707852 \\ Email: \\ Krzysztof.Murawski@wat.edu.pl
}

\author{
Artur Arciuch \\ Military University of Technology \\ ul. Kaliskiego 2, \\ 01-489 Warsaw, Poland, \\ Email: \\ Artur.Arciuch@wat.edu.pl
}

\author{
Tadeusz Pustelny \\ Department of Optoelectronics \\ Silesian University of Technology \\ ul. Krzywoustego 2, \\ 44-100 Gliwice, Poland \\ Email: \\ Tadeusz.Pustelny@polsl.pl
}

\begin{abstract}
The article presents new results achieved during researching the distance measuring method that is a part of Depth From Defocus techniques. The method has been developed to determine the shape of the flaccid diaphragm used in the Ventricular Assist Device (VAD). The shape is determined on the basis of distance measured between the CCD sensor plate of the camera and objects (markers) located on the flaccid diaphragm. Experiments were carried out using a stationary camera and circular markers with a diameter from $3 \mathrm{~mm}$ to $9 \mathrm{~mm}$.

The goal of this paper is to present the influence of the object (marker) size on the distance range measured between the camera and diaphragm used in the external pneumatic prosthetic heart.
\end{abstract}

\section{INTRODUCTION}

$\mathrm{T}$ HE ARTICLE presents the impact of the size of the observed object (marker) on the result of the distance measurement method presented in [1], [2]. This method is part of the group of techniques defined by the formulated Depth From Defocus (DFD). It was developed specifically for the video sensor determining the momentary volume of ejected blood $(S V)$ from the blood chamber of the pneumatic artificial heart. In [1], [2], the image is produced by a stationary camera equipped with a lens with a fixed focus. The camera is placed above the diaphragm so as to allow the observation of its entire surface. The proposed solution is characterized by the fact that during distance measurement the position of the camera and all lens and camera settings (focus, aperture, focal length) remain unchanged. Only the position of the observed markers located on the flaccid diaphragm is subject to change, Fig. 1a, which is located between the blood chamber and the air chamber, Fig. 1b. Method [1], [2] simultaneously determines the position of all of the markers in 3D space based on an analysis of only one image frame. In this respect, it has no equivalent in the literature. The vision systems, mentioned in the literature, used for measuring the distance consist of a light source and a camera, which usually form a stereoscopic system [3]. The distance in such systems is calculated by knowing the optical parameters of the cameras and their mutual position. Measuring systems equipped with one camera are also not rare [4]. The camera then performs two to eight photos of the object [4], and the distance is determined on the basis of the inverse perspective transformation [5]. There is also a measuring system variant with a camera equipped with autofocus. Such a system is calibrated with the help of a standard with known parameters. Measuring the distance to the object then depends on taking a picture at a specific focus setting and calculating the distance using the lens equation [6]. Other methods used to measure the distance is photogrammetry [7], [8] and the fringe projection technique [9] -[11]. Areas of application are, however, limited by the scope of the distances, the speed of the autofocus settings, image processing time, the resolution of the camera image sensor, the number of processed frames per second, as well as the dimensions and weight of the sensor.

In the application, considered in the article, real-time operation is particularly important with the simultaneous determination of the location of 49 markers located on the flaccid diaphragm in 3D space, Fig. 1a, which shape can change with the frequency of up to $3 \mathrm{~Hz}$.
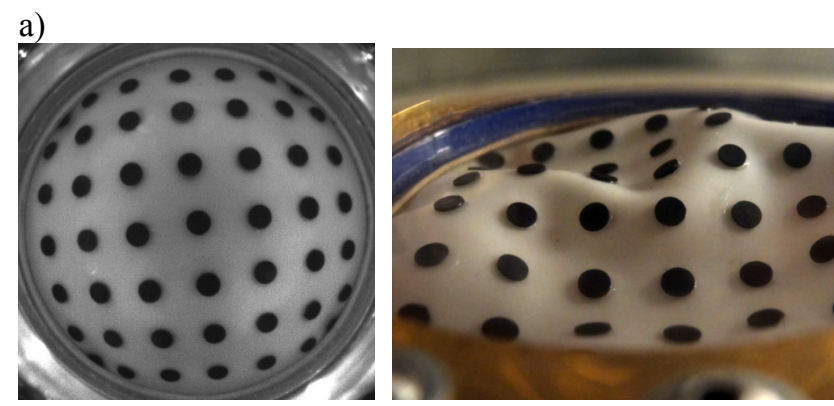

b)

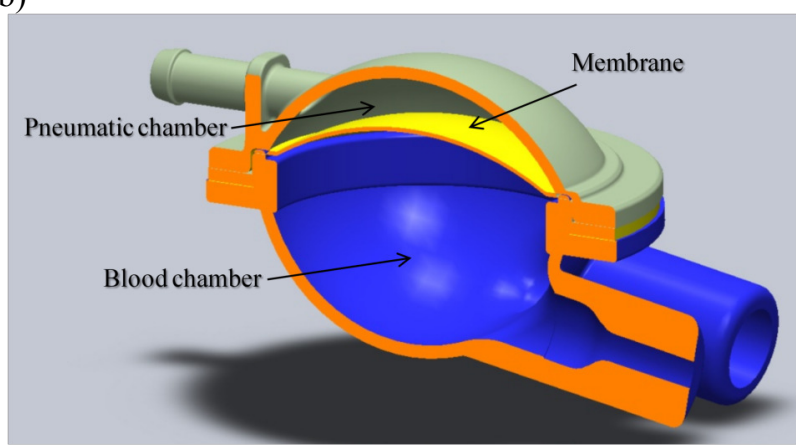

Fig. 1 View of flaccid diaphragm (a), location of the observed diaphragm in the artificial heart prosthetic (b) 


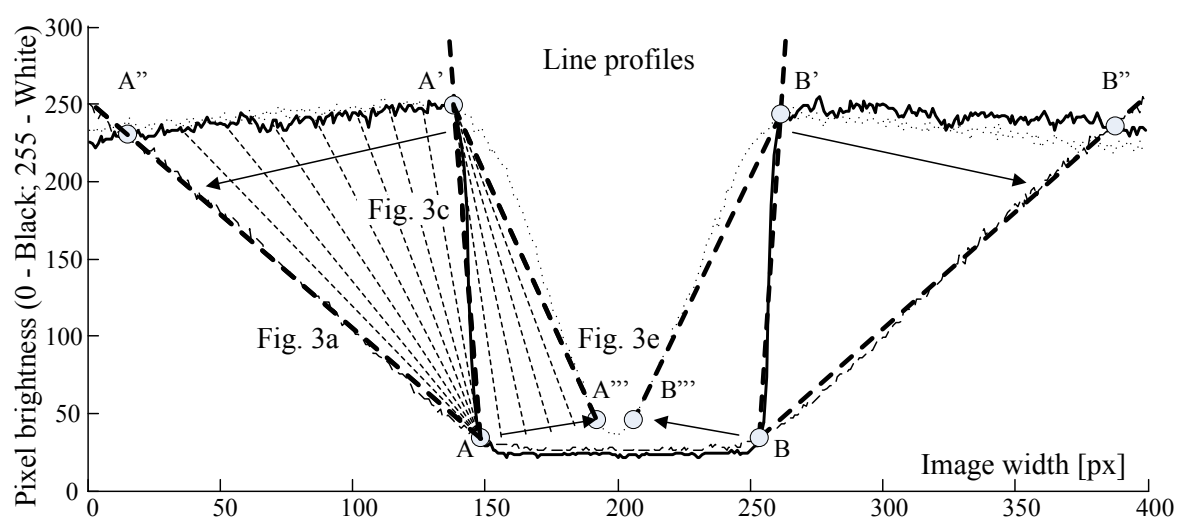

Fig. 2 Profile images of horizontal lines designated by the center of gravity of the object (the view on the standardization of brightness) [1]

\section{New DePth From Defocus Method [1], [2]}

The essence of the presented measurement solution is based on the analysis of the image produced by the marker moving away from its location for which its focus was set. Figure 2 shows the profiles of horizontal lines of images indicated by the center of gravity of the marker as well as their behavior during changes in the distance of the marker from the camera. For sharp views of marker lengths $\mathrm{AA}^{\prime}$ ' and BB' in Fig. 2 are almost vertical, which indicates a sharp cutoff of the view of the marker from the background. When the marker approaches the camera image blurs. As a result, points A' and B' move respectively in direction A" and B". At the same time the distance between points $\mathrm{A}$ and $\mathrm{B}$ remains practically unchanged. When the marker moves away from the camera points $A$ " and $B$ " return to their original position ( $\left.A^{\prime \prime} \rightarrow A^{\prime}, B^{\prime \prime} \rightarrow B^{\prime}\right)$, and the marker image becomes sharp. The marker further distancing from the camera results in a decrease in the view of the marker and the image blurs. In the case under consideration a displacement of points $\mathrm{A} \rightarrow \mathrm{A}$ "' i $\mathrm{B} \rightarrow \mathrm{B}$ "'is observed. The position of points $\mathrm{A}^{\prime}$ and $\mathrm{B}^{\prime}$ remains unchanged. The sequence of changes in the position of points $\mathrm{A}, \mathrm{A}^{\prime}, \mathrm{B}, \mathrm{B}$ ' determined when approaching and moving away from the marker with the camera at a step $\Delta L=0.01 \mathrm{~m}$ is presented in Fig. 3. Changes in positions of points A, A', B, B' shown in Fig. 2 and Fig. 3 are the basis for determining the distance marker. For this purpose, the image from the camera is subjected to defuzzification. Defuzzification was performed using image binarization with a threshold $\mathrm{T}_{\mathrm{H}}$ equal to 70 . The selection of the binarization threshold consists of determining such a $\mathrm{T}_{\mathrm{H}}$ value, so that a uniform distribution of intersecting points of horizontal image line profiles is obtained (indicated by the marker center of gravity) with a line showing the $T_{H}$ test value, Fig. 3. The location of the determined points univocally associated with distance $d$ of the marker to the plane of camera image, Fig. 3. The points distribution is described by the equation during the calibration process. The result is a relation describing the distance of the marker to the image plane of the camera.

\section{MOTIVATION}

The motivation to work on video sensor hardware and software (soft-sensors) to measure the $S V$ pneumatic pulsating heart supporting pump were the test results obtained in the framework of the "Polish Artificial Heart"

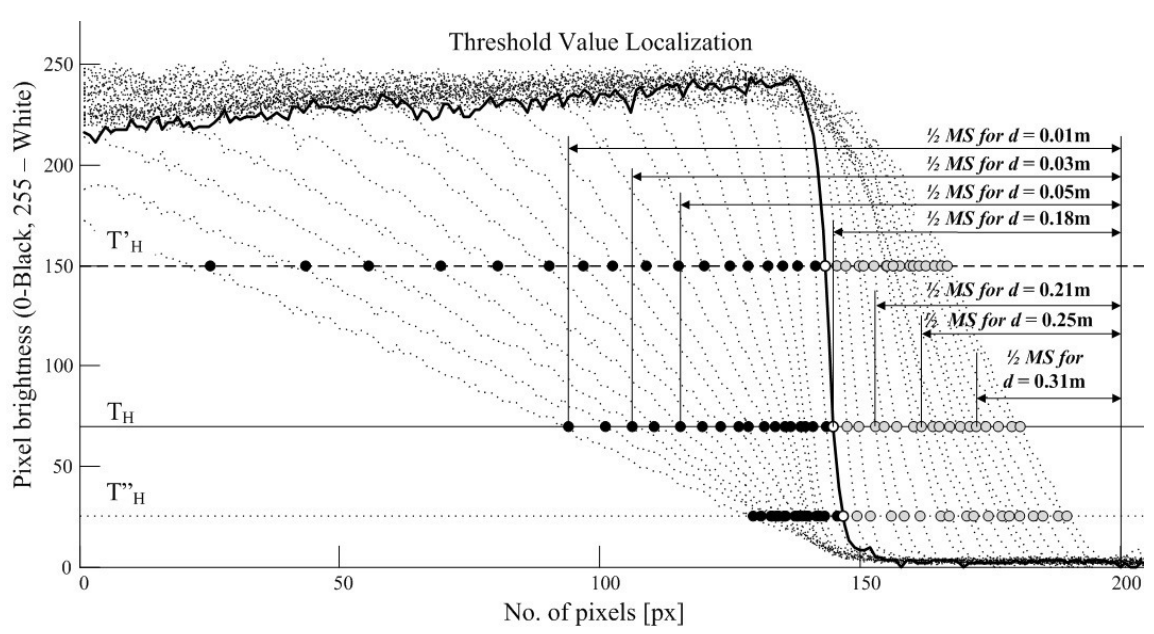

Fig. 3 The distribution of points relevant due to determining the distance to the marker for the established threshold $\mathrm{T}_{\mathrm{H}}$ at $L_{M I N}=0.07 \mathrm{~m}, L_{M A X}=0.42 \mathrm{~m}$ and $\Delta L=0.01 \mathrm{~m} \mathrm{[1]}$. 
project [12]. Both [13], [14] have demonstrated that the instantaneous stroke volume can be determined by an acoustic technique using the Helmholtz resonator theory. Knowing the limitations of the developed method the project also examined the possibility of using other techniques and measuring devices [15], including the video camera [16]. [16] shows that the measurement of momentary stroke blood volume of the ReligaHeart ${ }^{\circledR}$ EXT heart prosthesis using a video camera and a marker is ambiguous and burdened with error. The experiment described in [16] was verified by conducting a test using a model of the heart supporting pneumatic pump. In the test, just like in [16], occurrences of stroke volume measurements were noticed for which different shapes of the flaccid diaphragm were characterized with identical positions and marker areas, Fig. 4. This behavior of the marker prevents unambiguous determination of blood stroke volume only on the basis of analyzing changes in its position and the size of the surface area.

Using flaccid diaphragms in the heart prosthesis, even though raises many problems, is necessary and justified from a medical point of view. Such a diaphragm limits the formation of coagulation and eliminates the problem of sedimentation of blood (dividing into fractions). This does not change the fact that the problem of determining the momentary stroke volume of blood from the blood chamber of the pneumatic pump heart assist device (artificial heart) remains unresolved, and the safety of its use is based solely on its visual inspection: "One of the main advantages of the extracorporeal, polyurethane blood pump is its transparency that allows running continuous visual inspection of the pump status and its quality of work." [17] As an alternative to the present status it is proposed to use virtual reality technology in the sensor system of the chamber. In the method presented in [18], the camera captures a twodimensional image of the diaphragm equipped with passive markers, Fig. 5. Markers are used to determine characteristic points of the flaccid diaphragm in 3D space. Knowing the position of these characteristic points in [18] the method of reconstructing the view of the diaphragm was stated.

This method initialized on an IBM PC type computer allows calculating the coordinates of nodal points of the diaphragm and to generate the visualization in 3D space with a frequency of $7 \mathrm{~Hz}$. Exemplary results of the reconstruction

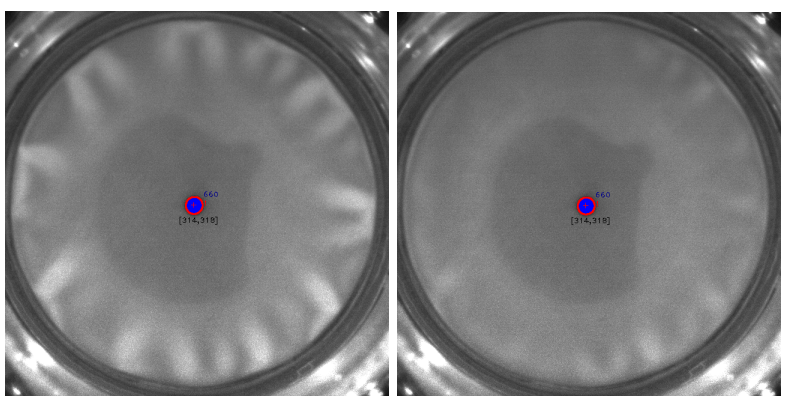

Fig. 4 Unambiguous measurement of SV resulting from the usage of one marker: marker position [314px,318px], surface equalling 660px

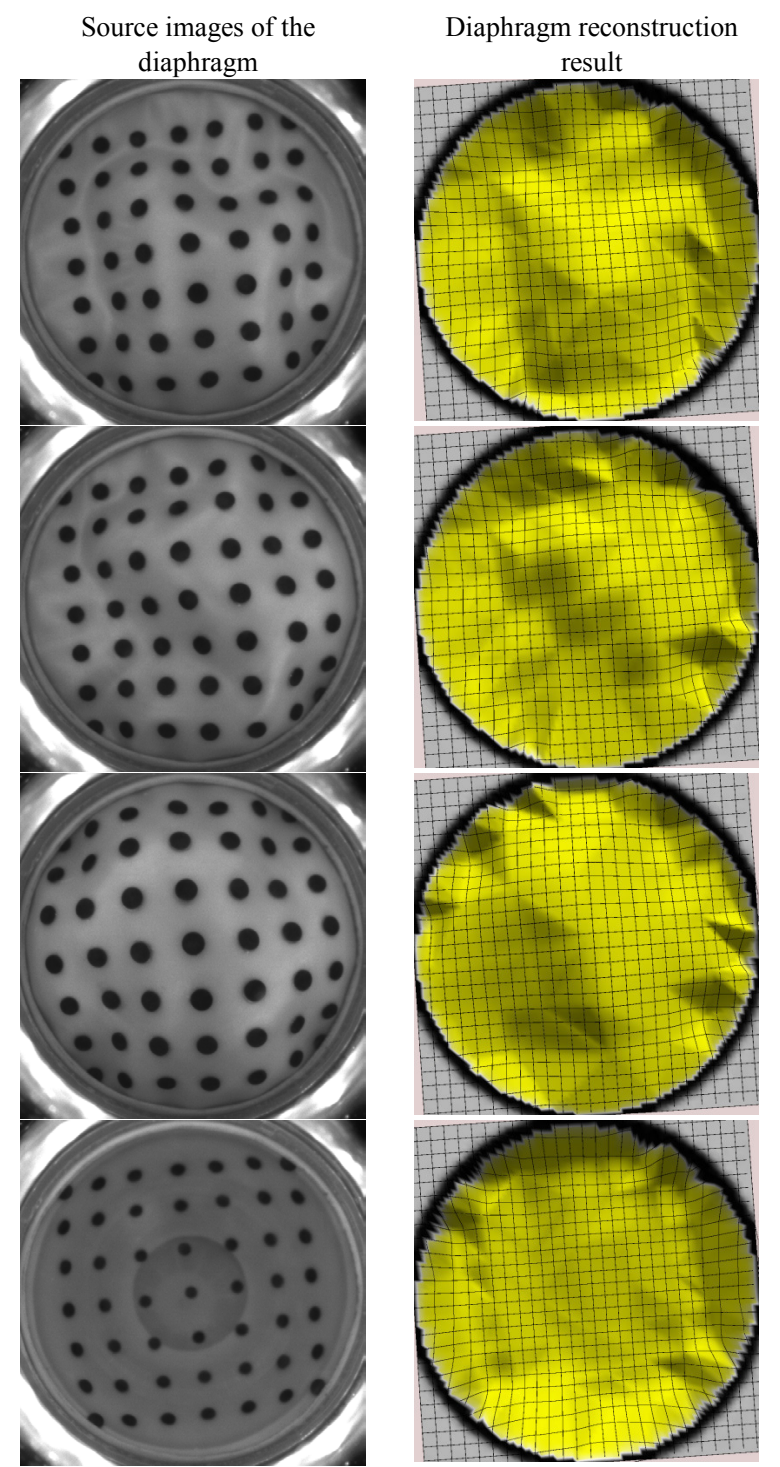

Fig. 5 Reconstructing the view of the flaccid diaphragm based on one photo using the technique provided in [18]

of the view of the diaphragm is shown in Fig. 5. The accuracy in the reconstruction, and thus determining the $S V$ depends on [1],[2] by the number and arrangement of the markers as well as the precision of the determined distances. For this reason, the article placed great emphasis on the examination of the influence of the marker size on the value of the determined distance. The acquired dependencies and recommendations to use method [1],[2] was included in part $\mathrm{V}$ and VI of this study.

\section{MEASUREMENT SYSTEM CONFIGURATION}

The influence of the size of the marker on the value of the determined distance was studied using the Optitrack v100: Slim camera. The camera was equipped with a tripod, a lens with a brightness of $F=2.0$ with a fixed focus of $f=16 \mathrm{~mm}$, visible light filter (high-pass filter, $\lambda \geq 850 \mathrm{~nm}$ ), infrared illuminator with the wavelength $\lambda=850 \mathrm{~nm}$ and a illuminator driver. The operation of the controller and the IR 


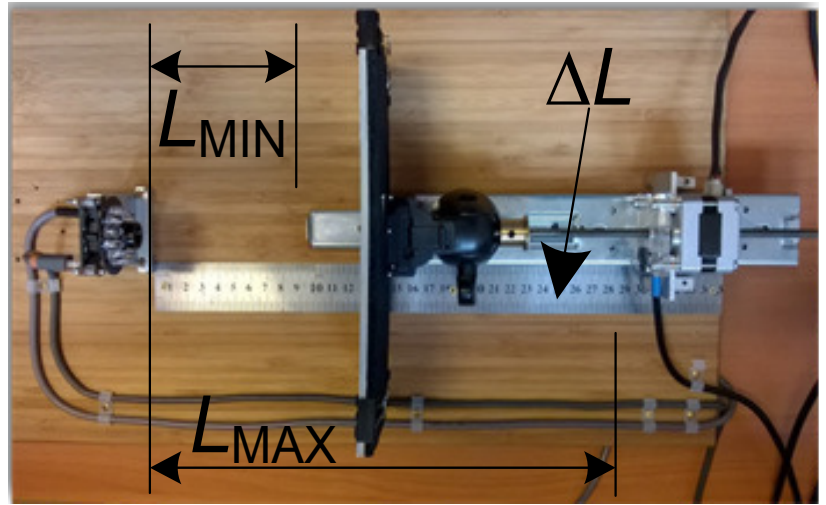

Fig. 6 View of the measuring system

lamp together are discussed in [19] - [21]. The experiment was performed in the configuration shown in Fig. 6. The distance marked in Fig. 6 equaled $L_{\mathrm{MIN}}=0.105 \mathrm{~m}$, $L_{\mathrm{MAX}}=0.175 \mathrm{~m}, \Delta L=0.001 \mathrm{~m}$. In the study, the focus of the lens was set to a distance of $L=0.14 \mathrm{~m}$, measuring from the image sensor plane of the camera. This position was taken as a reference point $d_{0}$ (zero position). All distance measurements were performed from this point. Measurements were performed for the range that included the permissible displacement of the front flaccid diaphragm occurring in the extracorporeal pneumatic heart prosthesis: $d_{0} \pm 0.035 \mathrm{~m}$. Studying the influence of the size of the marker area on the scope of measured distances was divided into two stages. The first stage consisted of acquiring images of marker views. Images were acquired in grayscale with a resolution of $640 p x \times 480 p x$. In the tests the distances were determined to the white plane, Fig. 6, bearing the marker. The marker was a black circle with a diameter from $0.003 \mathrm{~m}$ to $0.009 \mathrm{~m}$. Exemplary views of the marker along with the result of its processing are shown in Fig. 7. The position of the plane with the marker was determined by setting it relative to $d_{0}$ with an accuracy of $\pm 0.00001 \mathrm{~m}$. For this purpose a 39BYGL215A stepper motor was used. The engine with the pusher was placed on a crane. The tip of the

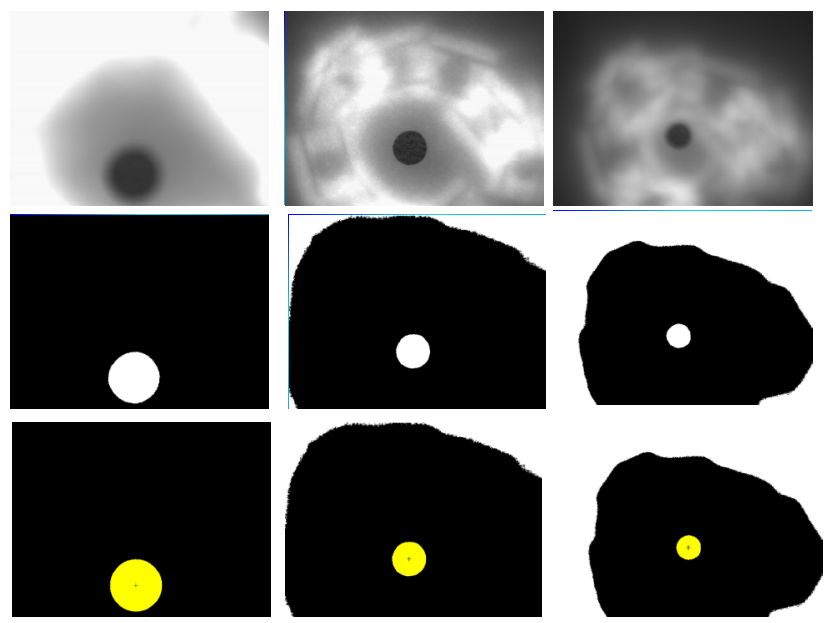

Fig. 7 Marker views (by rows): source image, image after segmentation, image result with the indicated center and analyzed marker surface pusher was attached to a movable plane, Fig. 6 . The straight edge was mounted parallel to the crane, which was used for visually checking the accomplishments of each push given.

The second stage consisted of carrying out activities related to the processing of raster images according to the method provided in [1],[2]. The analysis was then carried out with the aim of searching the connection existing between the initial value of the marker, the range of change observed on the surface of the marker and the obtained measurement range.

\section{RESULTS OF RESEARCH}

Studying the effect of the marker area size for distance measurement was performed for markers with a diameter of $0.003 \mathrm{~m}, 0.004 \mathrm{~m}, 0.005 \mathrm{~m}, 0.006 \mathrm{~m}, 0.007 \mathrm{~m}, 0.008 \mathrm{~m}, 0.009 \mathrm{~m}$. The experiment was performed in the configuration shown in Fig. 6. All distance measurements were carried out with the same lighting conditions. For each $i$-th marker the marker area was first defined in neutral position $d_{0}$. Then the screen was moved towards the camera with a step of $0.001 \mathrm{~m}$. This was repeated until achieving a displacement of $0.035 \mathrm{~m}$. The displacement was realized by a stepper motor controlled by a microcomputer system. For each of the predefined positions the marker made 100 measurements of its surface area. The test results were accepted as the arithmetic average determined from a series of measurements. After the completion of this experiment the screen with the marker would come back to the zero position. In the next step, measurements were carried out, in which the screen with the marker would move away from the camera image sensor plane. As before, the change in the area of the tested marker was recorded with a step of $0.001 \mathrm{~m}$. The last measurement was performed with removing the marker relative to the position for which the sharpness was fixed at $0.035 \mathrm{~m}$. These actions were implemented for all seven markers tested. The results are presented in Table I. The surface areas of the markers presented in Table I were determined through operations on the image. For this purpose, for each measurement the following was carried out:

* image defuzzification;

* identifying the location of the spot on the image that matches the location of the marker;

* identifying the location of the center of the marker;

* determining the area of the marker in pixels.

The range of measured distances, in the studied DFD technique, is due to the nature of changes in the surface area of the marker view visible in the image after defuzzification. The resulting variability of the surface areas of the markers in the measuring range of $d 0 \pm 0.035 \mathrm{~m}$ is shown in Fig. 8. It shows that the least variability of $2383 \mathrm{px}$ (reference value), was obtained for the marker with a diameter of $0.003 \mathrm{~m}$. The marker with the given diameter, although most promising due to the possibility of a high density of markers on the diaphragm surface does not give the basis (only based on the 
TABLE I.

THE MEASUREMENT RESULTS OF THE MARKER SURFACE FOR THE DISTANCE RANGE OF $D_{0} \pm 0.035 \mathrm{M}$ WITH A STEP OF 0.005M

\begin{tabular}{|c|c|c|c|c|c|c|c|c|c|c|c|c|c|c|}
\hline & \multicolumn{14}{|c|}{ Displacement in relation to position zero in [m] } \\
\hline & 0.035 & -0.030 & -0.025 & -0.020 & -0.015 & -0.010 & -0.005 & 0.005 & 0.010 & 0.015 & 0.020 & 0.025 & 0.030 & 0.035 \\
\hline $0.003 \mathrm{~m}$ & 3598 & 3397 & 3170 & 2922 & 2682 & 2449 & 2247 & 1934 & 1806 & 1685 & 1569 & 1449 & 1334 & 1215 \\
\hline 0.00 & 93 & 70 & 746 & 525 & 87 & & 3 & 3443 & 3224 & 7 & 6 & 98 & 7 & 22 \\
\hline 0.005 & 334 & 9495 & 8706 & 7954 & 7263 & 6633 & 6090 & 246 & 914 & 5 & 5 & 066 & 8 & 3561 \\
\hline $0.006 \mathrm{~m}$ & 4431 & 13239 & 12122 & 11106 & 10187 & 9356 & 8619 & 7402 & 6897 & 6424 & 5988 & 5571 & 5171 & 4785 \\
\hline $0.007 \mathrm{~m}$ & 19719 & 18077 & 16556 & 15174 & 13910 & 12789 & 11778 & 10109 & 9442 & 8823 & 8249 & 7716 & 7214 & 6735 \\
\hline $0.008 \mathrm{~m}$ & 25647 & 23454 & 21464 & 19681 & 18064 & 16611 & 15313 & 13095 & 12175 & 11322 & 10526 & 9778 & 9088 & 8454 \\
\hline $.009 \mathrm{~m}$ & 39891 & 36419 & 33311 & 30594 & 28147 & 25936 & 23935 & 20496 & 19033 & 17721 & 16533 & 15442 & 14480 & 3593 \\
\hline
\end{tabular}

A displacement equal to zero was registered for markers: 2070, 3707, 5632, 7962, 10894, 14156, 22122.

knowledge of the measured surface area) to perform an accurate distance measurement. The change in the surface marker with a diameter of $0.003 \mathrm{~m}$ is determined as a function of distance dependence $f(x)=6.82 x^{2}-279.42 x+$ 3906.4. A similar result was observed for the marker with a diameter of $0.004 \mathrm{~m}$ and $0.005 \mathrm{~m}$. The variability of these markers equaled 4571px (increase of approx. 2 times) and 6773px (increase of approx. 3 times) accordingly, and the nature of changes are defined by dependencies $f(x)=16.39 x^{2}$ $579.83 \mathrm{x}+7326.20$ for the $0.004 \mathrm{~m}$ diameter marker and $f(x)=$ $26.81 x^{2}-898.10 x+11,148$ for the $0.005 \mathrm{~m}$ diameter marker. Better quality results were obtained for markers with a diameter of $0.006 \mathrm{~m}, 0.007 \mathrm{~m}, 0.008 \mathrm{~m}$ and $0.009 \mathrm{~m}$. The nature of the changes in their surface areas are defined by third degree polynomials: $f(x)=-1.54 x^{3}+70.44 x^{2}-1,445.7 x+$ 15,841 for $0.006 \mathrm{~m} ; f(x)=-1.93 x^{3}+93.99 x^{2}-1,966.5 x+$ 21,631 for $0.007 \mathrm{~m} ; f(x)=-2.57 x^{3}+120.74 x^{2}-2,541 x+$ 28,071 for $0.008 m$ and $f(x)=-3.56 x^{3}+179.39 x^{2}-3,887 x+$ 43,535 for $0.009 \mathrm{~m}$. For the given markers change ranges were determined: 9,646px (increase approx. 4 times), $12,984 \mathrm{px}$ (increase approx. 5.5 times) 17,193px (increase of approx. 7 times) and 26,298px (increase of approx. 11 times). The obtained results show that the best of the tested markers to measure distance was the marker with a diameter of $0.009 \mathrm{~m}$. The marker with such a diameter, however, is not suitable for use in the task of determining the shape of the diaphragm (flaccid diaphragm) of a pulsatile pneumatic heart assist pump. Due to its size it does not provide the assurance of performing a high density of markers on the surface of the diaphragm, in order to precisely reproduce its shape in a computerized measurement system. Accurate projection of

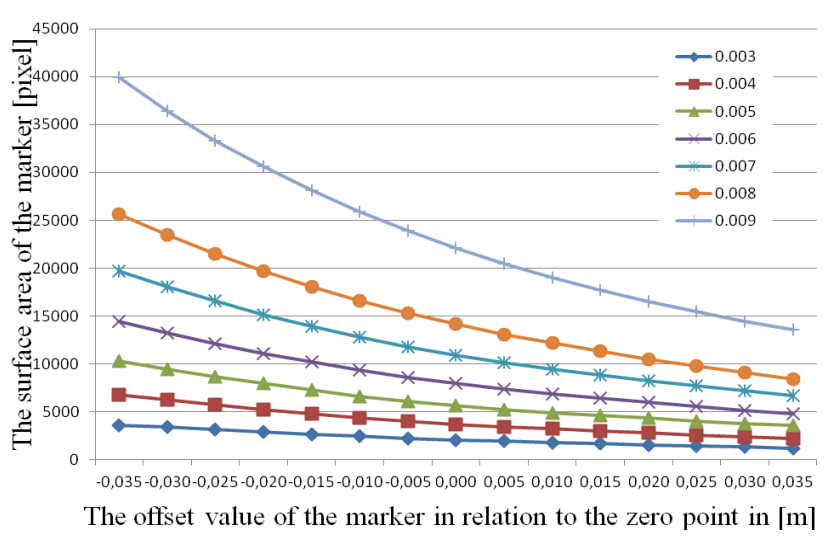

Fig. 8 Measurement results on the variability of surface areas of the markers

the diaphragm shape requires displaying the greatest possible number of markers on its surface, Fig. 5. Hence the simple conclusion that the markers should be as small as possible. Therefore, the analysis of the test results were repeated. At the time it was noticed that the implementation of the standardization of the results with respect to the surface area of the marker captured at point $d_{0}$ introduces a significant change in the acquired variable ranges of the marker surface areas, Table II. The graphic visualization of the results are shown in Fig. 9. As a result of carrying out the standardization of the results it turned out that the diameter of the marker had no significant impact on the accuracy of the distance measurement being performed. The shape of the "Mean Value" curve presented in Fig. 10 was described by the polynomial $f(x)=-0.025 x^{3}+0.124 x^{2}-0.238 x+0.140$, where $x$ is the normalized surface area of the marker.

TABLE. II.

THE MEASUREMENT RESULTS OF THE MARKER SURFACE FOR THE DISTANCE RANGE OF $D_{0} \pm 0.035 \mathrm{M}$ WITH A STEP OF 0.005M AFTER STANDARDIZATION

\begin{tabular}{|c|c|c|c|c|c|c|c|c|c|c|c|c|c|c|}
\hline & \multicolumn{14}{|c|}{ Displacement in relation to position zero in [m] } \\
\hline & -0.035 & -0.030 & -0.025 & -0.020 & -0.015 & -0.010 & -0.005 & 0.005 & 0.010 & 0.015 & 0.020 & 0.025 & 0.030 & 0.035 \\
\hline $0.003 \mathrm{~m}$ & 1.738 & 1.641 & 1.531 & 1.411 & 1.295 & 1.183 & 1.085 & 0.934 & 0.872 & 0.814 & 0.758 & 0.700 & 0.644 & 0.587 \\
\hline $0.004 \mathrm{~m}$ & 1.832 & 1.691 & 1.550 & 1.416 & 1.291 & 1.178 & 1.082 & 0.928 & 0.869 & 0.811 & 0.754 & 0.700 & 0.649 & 0.599 \\
\hline $0.005 \mathrm{~m}$ & 1.834 & 1.685 & 1.545 & 1.412 & 1.289 & 1.177 & 1.081 & 0.931 & 0.872 & 0.819 & 0.769 & 0.721 & 0.676 & 0.632 \\
\hline $0.006 \mathrm{~m}$ & 1.812 & 1.662 & 1.522 & 1.394 & 1.279 & 1.175 & 1.082 & 0.929 & 0.866 & 0.806 & 0.752 & 0.699 & 0.649 & 0.601 \\
\hline $0.007 \mathrm{~m}$ & 1.810 & 1.659 & 1.519 & 1.392 & 1.276 & 1.173 & 1.081 & 0.927 & 0.866 & 0.809 & 0.757 & 0.708 & 0.662 & 0.618 \\
\hline $0.008 \mathrm{~m}$ & 1.811 & 1.656 & 1.516 & 1.390 & 1.276 & 1.173 & 1.081 & 0.925 & 0.860 & 0.799 & 0.743 & 0.690 & 0.642 & 0.597 \\
\hline $0.009 \mathrm{~m}$ & 1.803 & 1.646 & 1.505 & 1.383 & 1.272 & 1.172 & 1.082 & 0.926 & 0.860 & 0.801 & 0.747 & 0.698 & 0.654 & 0.614 \\
\hline
\end{tabular}

For a displacements equaling zero all sizes of determined surface markers took on the value of 1 . 


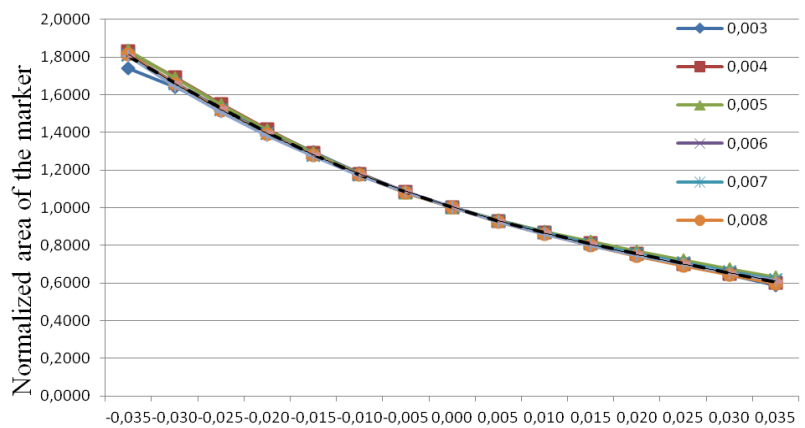

The offset value of the marker in relation to the zero point in $5 \mathrm{ml}$

Fig. 9 The measurement results after normalization

For the given equation the average distance measurement error did not exceed $\pm 0.00035 \mathrm{~m}$.

\section{CONCLUSION}

The article includes the course and results of the new DFD technique [1],[2]. The purpose of the experiments was to determine the influence of the marker on the result and the scope of the measured distance. Tests were carried out for seven markers with a diameter of $0.003 \mathrm{~m}$ to $0.009 \mathrm{~m}$.

The method of measuring the distance presented in [1],[2] have been developed to determine, in real time, the shape of the flaccid diaphragm [18] of the pulsating pneumatic heart assist pump (artificial heart), Fig. 5. Therefore, it was particularly important to evaluate the effect of the measurement method in the range of movements that the diaphragm is subject to in the prosthetic heart model.

The achieved results show that in the range of $\mathrm{d} 0 \pm 0.035 \mathrm{~m}$ the tested technique allows to obtain high accuracy measurements. The measurement error during the test did not exceed $\pm 0.00035 \mathrm{~m}[1]$.

Based on achieved results the following recommendations were formulated:

1) where the standardization is not used it is recommended to use markers with a diameter no less than $0.006 \mathrm{~m}$;

2) the normalization of the determined surface area of the marker enables the use of markers having a diameter of no less than $0.006 \mathrm{~m}$.

3 ) in order for the distance measurement results to be independent from the diameter of the marker it is recommended to use the standardization of the marker surface area and determine the distance to the object from the polynomial $f(x)=-0.025 x^{3}+0.124 x^{2}-0.238 x+0.140$, where $x$ is the standardized surface area of the marker.

\section{REFERENCES}

[1] K. Murawski, "Method of Measurement the Distance to an Object Based on One Shot Obtained from a Motionless Camera with a FixedFocus Lens", Acta Physica Polonica A, 127, 6, pp. 1591 - 1595, 2015. http://dx.doi.org/10.12693/APhysPolA.127.1591

[2] K. Murawski, "Method of measuring the distance using the cameras", Patent Application No. P.408076, 2014, (in Polish).

[3] H. Wang, J. Hu, "Active stereo method for three - dimensional shape measurement", Optical Engineering, 51, 6, pp. 1 - 8, 2012. http://dx.doi.org/10.1117/1.OE.51.6.063602
[4] SY. Chen, YF. Li, "Finding Optimal Focusing Distance and Edge Blur Distribution for Weakly Calibrated 3-D Vision", IEEE Transactions on Industrial Informatics, 9, 3, pp. 1680-1687, 2013. http://dx.doi.org/10.1109/TII.2012.2221471

[5] F. Bonin-Font, A. Burguera, A. Ortiz, G. Oliver, "A Monocular Mobile Robot Reactive Navigation Approach Based on the Inverse Perspective Transformation", ROBOTICA, 31, pp. 225 - 249, 2013. http://dx.doi.org/10.1017/S0263574712000252

[6] A. de La Bourdonnaye, R. Doskočil, V. Křivánek, "Practical Experience with Distance Measurement Based on Single Visual Camera", Advances in Military Technology, 7, 2, 49 - 56, 2012.

[7] http://tdserver1.fnal.gov/darve/mu_cool/pressuretest/Basics_of_Photo grammetry.pdf, (2015).

[8] K. Yue, Z. Li, M. Zhang, S. Chen, "Transient full-field vibration measurement using spectroscopical stereo photogrammetry", OPTICS EXPRESS, 18, no. 26, pp. 26866 - 26871, 2010. http://dx.doi.org/10.1364/OE.18.026866

[9] Y. Morimoto, A. Masaya, M. Fujigaki, D. Asai, Applied Measurement Systems, chapter 7, 137, ISBN 978-953-51-0103-1, 2012.

[10] R. B. Rusu, A. Aldoma， S. Gedikli, M. Dixon, "3D Point Cloud Processing: PCL", Tutorial at IEEE/ RSJ International Conference on Intelligent Robots and Systems (IROS), 2011.

[11] A. Saxena, H. Koppula, R. Newcombe, X. Ren, "RGB-D: Advanced Reasoning with Depth Cameras", Workshop in conjunction with Robotics: Science and Systems (RSS), 2013.

[12] Red.: J. Sarna, R. Kustosz, E. Woźniewska, M. Gonsior, A. Jarosz, K. Szymańska, D. Hansel, E. Krzak, "Program Polskie Sztuczne Serce", ISBN 978-83-63310-16-5, 2013.

[13] T. Pustelny, G. Konieczny, Z. Opilski, M. Gawlikowski, "Measuring systems for pulsatile heart assist pumps ReligaHeart ${ }^{\circledR}$ - measuring system movement of the diaphragm", Polish artificial heart, the development of design, qualification tests, preclinical and clinical, ISBN 978-83-63310-12-7, pp. 22 - 36, 2013, (in Polish).

[14] P. Gibinski, G. Konieczny, E. Maciak, Z. Opilski, T. Pustelny, "Acoustic device for measuring instantaneous blood volume in cardiac support chamber i.e. pneumatic heart assist driving chamber, has sensor supporting heart in openings, and audio amplifier connected with volume unit of blood-cell support", Patent No. PL394074-A1.

[15] G. Konieczny, T. Pustelny, P. Marczyński, "Quasi-Dynamic Testing of an Optical Sensor for Measurements of the Blood Chamber Volume in the POLVAD Prosthesis", Acta Physica Polonica A, 124, 3, pp. 483-485, 2013. http://dx.doi.org/10.12693/APhysPolA.124.483

[16] D. Komorowski, M. Gawlikowski, "Preliminary investigations regarding the blood volume estimation in pneumatically controlled ventricular assist device by pattern recognition", Computer recognition systems 2, ASC 45, pp. 558 - 565, 2007. http://dx.doi.org/10.1007/978-3-540-75175-5_70

[17] R. Kustosz, A. Jarosz, M. Gawlikowski, A. Kapis, M. Gonsior, "The role and perspectives of development of the Polish air pump heart assist on the market of heart prosthetic", Polish artificial heart, the development of design, qualification tests, preclinical and clinical, ISBN 978-83-63310-12-7, 2013, (in Polish).

[18] K. Murawski, T. Pustelny, M. Murawska, "System and method of determining the shape of diaphragm of pneumatic extracorporeal heart assist pump", Patent Application No. P.414104, 2015, (in Polish).

[19] K. Murawski， D. Białas， M. Rękas, "Measurement of Corneal Neovascularisation with the use of Image Processing Techniques", Acta Physica Polonica A, vol. 127, 6, pp. 1732 - 1736, 2015. http://dx.doi.org/10.12693/APhysPolA.127.1732

[20] K. Różanowski, K. Murawski, “An Infrared Sensor for Eye Tracking in a Harsh Car Environment", Acta Physica Polonica A, 122, 5, pp. $874-879,2012$. http://dx.doi.org/10.12693/APhysPolA.122.874

[21] K. Murawski, R. Różycki, P. Murawski, A. Matyja, M. Rekas, An Infrared Sensor for Monitoring Meibomian Gland Dysfunction, Acta Physica Polonica A, 124, 3, 517 - 520, 2013. http://dx.doi.org/10.12693/APhysPolA.124.517 